\title{
Non-alcoholic fatty liver disease (NAFLD) and immune response
}

\author{
${ }^{1}$ Adhikari, Khem Bahadur and ${ }^{2}$ Tirosh Oren \\ ${ }^{1}$ Department of Food Sciences, Institute for Technology and Storage of Agricultural Products, Agricultural Research \\ Organization, The Volcani Center, Bet Dagan, Israel \\ ${ }^{2}$ Institute of Biochemistry, Food Science and Nutrition, The Robert H. Smith Faculty of Agriculture, Food and Environment, \\ The Hebrew University of Jerusalem, Rehovot, Israel
}

Correspondence to: Khem Bahadur Adhikari, Division for External Studies, The Robert H. Smith Faculty of Agriculture, Food and Environment, The Hebrew University of Jerusalem, Israel

Email:khem-bah.adhikari@mail.huji.ac.il

\begin{abstract}
Introduction: Non-alcoholic fatty liver disease (NAFLD) is a chronic liver disease that affects a high proportion of world's population. This review aims to update latest advances in epidemiology, clinical aspects, pathogenesis, mechanism, immune responses and therapies of NAFLD.

Methods: This paper was prepared with reviews of published articles and books.

Results: NAFLD, the commonest liver disorder in the world, is closely associated with metabolic syndrome, particularly obesity and insulin resistance. Sedentary lifestyle and poor dietary choices are leading to obesity, subsequently increasing risk for developing metabolic syndrome and NAFLD. NAFLD is characterized with increased lipogenesis, defective mitochondrial and peroxisomal boxidation and/or disability to lipid export from the liver. It can progress to cirrhosis, hepatocellular carcinoma, and liver failure. The development and progression of NAFLD are also affected by diet and genetic factors. Poor immune system cannot overcome proinflammatory cytokines and reactive oxygen species (ROS) which cause oxidative liver injury. Up regulation of perioxisome proliferator activated receptor (PPAR) and disruption of sterol regulatory element binding protein (SREBP) are important targets for pharmacological intervention accompanied with lifestyle modifications.
\end{abstract}

Conclusions: People with obesity, type 2 diabetes mellitus (T2DM), and metabolic syndrome are likely to suffer from NAFLD.

Keywords: Cytokine, inflammation, NAFLD, obesity

\section{Introduction}

Non-alcoholic fatty liver disease (NAFLD) refers to the presence of hepatic steatosis not associated with a significant intake of alcohol ${ }^{1}$ and its incidence is paralleling the increasing numbers of overweight and obese individuals worldwide. ${ }^{2}$ When fat accounts for more than $10 \%$ of liver's weight, then the condition is called fatty liver and it can develop more serious complications. ${ }^{3}$ Fatty liver may cause no damage, but sometimes the excess fat leads to 
inflammation causing liver damage, called steatohepatitis. ${ }^{3}$ The term nonalcoholic steatohepatitis (NASH) was first coined by Ludwig et $\mathrm{al}^{4}$ describing the pathology of 20 patients histologically similar with alcoholic hepatitis but without the history of alcohol abuse. Sometimes, inflammation from a fatty liver is linked to alcohol abuse; this is known as alcoholic steatohepatitis. ${ }^{5,6}$ Otherwise the condition is called NASH. ${ }^{3}$ NAFLD comprises a spectrum of liver pathology including bland steatosis, steatohepatitis, cirrhosis $^{7}$ and hepatocellular carcinoma ${ }^{8}$ where most liver related morbidity and mortality occur. The histological damage in NAFLD is very similar to that seen in patients with ALD, but NAFLD is by definition not alcohol induced. ${ }^{8}$

NAFLD is the most common chronic liver disease in the western world. ${ }^{1}$ Sedentary lifestyle and poor dietary choices are leading to a weight gain epidemic in westernized countries, subsequently increasing the risk for developing the metabolic syndrome and NAFLD. ${ }^{9}$ NAFLD may be categorized as primary and secondary depending on the underlying pathogenesis (Table 1).

Table 1. Type and causes of NAFLD ${ }^{1,8}$

\begin{tabular}{|c|c|}
\hline Type & Causes/Associations \\
\hline Primary & $\begin{array}{l}\text { Obesity, glucose intolerance, hypertension, } \\
\text { hypertriglyceridemia, Low HDL cholesterol, } \\
\text { type } 2 \text { diabetes }\end{array}$ \\
\hline Secondary & $\begin{array}{l}\text { Drugs: corticosteroids, tamoxifen, diltiazem, } \\
\text { aspirin, highly active antiretroviral therapy, } \\
\text { valporate, amiodarone, estrogen, methotrexate, } \\
\text { zidovudine, tetracycline, cocaineInfections: } \\
\text { hepatitis C, human immunodeficiency viruses, } \\
\text { small bowel diverticulosis with bacterial } \\
\text { growth, endotoxinsMetabolic conditions: } \\
\text { Hypobetalipoproteinemia, lipodystrophy, } \\
\text { hypopitutarism, hypothalamic obesity, Weber } \\
\text { Christian syndrome, acute fatty liver of } \\
\text { pregnancy, Reyes syndromeToxins: organic } \\
\text { chemicals (petrochemicals), mushroom toxins } \\
\text { (Amanita phalloides), Bacillus cereus toxin, } \\
\text { EndotoxinsNutritional: Rapid weight loss, } \\
\text { intestinal bypass surgery, starvation, total } \\
\text { parental nutrition }\end{array}$ \\
\hline
\end{tabular}

\section{Epidemiology}

NAFLD is increasingly being recognized as an important and common condition, affecting approximately $20 \%$ of the general population in different countries (Table 2).$^{10}$ It is estimated to affect approximately $30 \%$ of the general US population and is considered the hepatic manifestation of the metabolic syndrome..$^{9,11}$ NAFLD affects one in three adults and one in 10 children in the United States. ${ }^{8}$ Although
NAFLD typically occurs between the fourth and six decades of life $\mathrm{e}^{12,13}$, it is known to affect children as well as adults and is not considered discriminatory to age. ${ }^{13,14}$ Many studies have found a wide discrimination of NAFLD between the sexes (Table 2). ${ }^{15,16,17}$

Among different ethnic groups, however, the picture becomes a bit more complicated. It has been reported that the prevalence of fatty liver was highest in Hispanics (45\%) compared to Caucasians (33\%) or African Americans $(24 \%) .{ }^{18}$ Furthermore, within specific race, such as Caucasians, sex-related differences in the presence of fatty liver ( $42 \%$ in men and $24 \%$ in women) had been observed, indicating race and sex related variability in the susceptibility to NAFLD. ${ }^{18}$ Among 3543 peoples, recently surveyed in South China, $609(17.2 \%)$ were diagnosed having fatty liver disease (FLD, 23.0\% in urban and $14.5 \%$ in rural) out of which prevalence of NAFLD was $15.5 \% .{ }^{13}$ In the same study, prevalence of FLD among the children at the age of 7-18 years was $1.3 \%$ with all having NAFLD. ${ }^{13}$ The prevalence and incidence of NAFLD is expected to increase worldwide as the global obesity epidemic spreads and the trend in developing countries toward the western lifestyle continues. $^{8}$

Table 2. Prevalence of fatty liver disease among adults

\begin{tabular}{|lllllllll|}
\hline Year Study & Country & N & Diagnosis Techniques & Prevalence (\%) \\
& & & & & & Overall & M & F \\
2007 & Amarapurkar et al; & India & 1168 & NAFLD & US & 16.6 & 24.6 & 13.6 \\
2006 Zelber-Sagi et al; & Israel & 352 & NAFLD & US & 30 & 21 & 38 \\
2006 Park et al; & Korea & 6648 & NAFLD & US & 16.1 & 21.6 & 11.2 \\
2007 Zhou et al; & China & 3543 & FLD & US & 17.2 & 20.6 & 27.6 \\
2004 Browning et al; & USA & 2287 & FLD & MRS & 34 & 42 & 24 \\
\hline
\end{tabular}

Notes: US, Ultrasonography; MRS, Magnetic Resonance Spectroscopy; NAFLD, Nonalcoholic fatty liver disease; FLD, Fatty liver disease; N, number of subjects; M, male; F, female

\section{Clinical aspects of NAFLD}

Most patients with NAFLD have no symptoms or signs of liver disease at the time of diagnosis. ${ }^{19}$ NAFLD has been characterized with asymptomatic elevation of aminotransferases, radiological findings of fatty liver or unexplained persistent hepatomegaly. ${ }^{19}$ NAFLD patients may be complaint of fatigue or a sensation of fullness or discomfort in the right upper abdomen. ${ }^{8}$ Hepatomegaly is one of the more consistent physical findings, described in up to $75 \%$ of patients with NAFLD. ${ }^{2}$ Other findings on physical examination that may suggest NAFLD as the cause 
of liver abnormalities include those characterizing insulin resistance and metabolic syndrome, such as central obesity, hypertriglyceridemia, and hypertension. ${ }^{2}$

The most common and often the only laboratory abnormality found in NAFLD patients, is mild to moderate elevation of liver enzymes ${ }^{8,19}$ alanine aminotransferase (ALT) and aspartate aminotransferase (AST): defined as ALT $>45$ U/L, AST>45 U/L or ã Glutamyl transferase (GGT) $>50 \mathrm{U} / \mathrm{L} .{ }^{20}$ In the patients with FLD, AST/ALT ratio is usually less than one, but this ratio increases as fibrosis advances. ${ }^{8}$ A study on Japanese adults showed that triglycerides, total protein albumin, AST and ALT were all significantly higher while high density lipoprotein (HDL) cholesterol and AST/ALT ratio were significantly lower in subjects with NAFLD than those without fatty liver. ${ }^{21}$

\section{Pathogenesis}

The primary metabolic abnormality leading to lipid accumulation in hepatocytes is not clearly understood however, it could potentially result from insulin resistance and alterations in lipid metabolism. ${ }^{19}$ Hepatic triglyceride accumulates when lipid influx and de novo synthesis exceeds hepatic lipid export and utilization. ${ }^{1}$ Hepatic steatosis is caused by the following metabolic disturbances: a) an increase in mobilization of fatty acids from adipose tissue, b) an increase in hepatic synthesis of de novo fatty acids, c) a decrease in fatty acid oxidation, d) an increase in triglyceride production, and e) a trapping of triglycerides in the liver. ${ }^{22}$ Excess production of cytokines, ROS, and the shortage of protective mediators, including adiponectin, play a role in pathogenesis of liver injury.

Currently the most widespread and prevailing theory for pathogenesis is the 'two-hit model', introduced by. ${ }^{23}$ The first hit involves the interference in the metabolism of free fatty acids (FFA) and triglycerides (TG) that usually accumulate in the liver causing steatotic liver. ${ }^{23}$ Accumulation of FFA supports a lipotoxicity mechanism for liver injury. ${ }^{24}$ In response to the excess fat in the liver FFA are oxidized and metabolized releasing ROS, cytokines and adipokines and exposing liver to oxidative stress, the second hit that transforms steatosis to steatohepatitis to fibrosis. ${ }^{2}$ Process of liver steatosis development is a continuous cycle (Fig. 1) that is fueled by the dietary intake and lifestyle of individuals. ${ }^{2}$

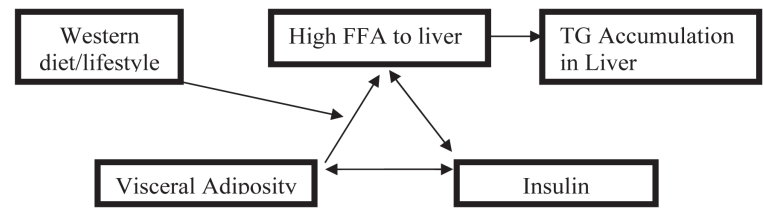

Fig. 1. Development of liver steatosis.

\section{Mechanisms and immune response}

The mechanisms underlying disease development and progression are insulin resistance, obesity related inflammation, genetic, dietary and lifestyle factors. ${ }^{11}$

\section{Oxidative and nitrosative stress}

An increase in intrahepatic concentration of FFA may provide a source of oxidative stress by peroxisomal boxidation, which unlike mitochondrial b-oxidation produces hydrogen peroxide $\left(\mathrm{H}_{2} \mathrm{O}_{2}\right)$ and this is again converted to highly reactive hydroxyethyl free radical in the presence of free iron. ${ }^{23}$ Increased systemic nitrosative stress with greater plasma nitrotyrosine (biomarker of nitrosative stress), and the oxidative stress predict the presence of NAFLD even in the absence of insulin resistance, metabolic syndrome, and hypoadiponectinemia, correlate with the severity of NAFLD.$^{25}$ In addition to creating oxidative stress, ROS can trigger other hits to catalyze disease progression. ${ }^{2}$ The other mechanism causing increased oxidative stress is lipid peroxidation of the increased FFA that accumulates in the first hit. ${ }^{2}$ The patients with NASH were found to have lower hepatic ù- 3 and ù- 6 polyunsaturated fatty acids (PUFAs) and decreased ratio of metabolites to essential fatty acids as compared to simple steatosis. ${ }^{26}$ This should be the outcome of lipid peroxidation which often affects PUFAs due to multiple double bonds. In non alcoholics, the cytochrome P450 enzyme (CYP2E1) can also generate free radicals from endogenously produced ketones and aldehydes, and dietary $\mathrm{N}$-nitrosamines causing oxidative stress. $^{23}$

\section{Adipocytokines and immune reaction}

The liver and the adipose tissue are the major sources of inflammatory mediators, termed adipocytokines, in human and experimental animals. ${ }^{7}$ Different adipocytokines and their role in NAFLD have been shown below (Table 3 ).

Table 3. Inflammatory mediators (adipocytokines) and their role in $\mathrm{NAFLD}^{7}$

\begin{tabular}{|c|c|}
\hline $\begin{array}{l}\text { Inflammatory } \\
\text { mediators }\end{array}$ & Role in NAFLD \\
\hline TNF-á & $\begin{array}{l}\text { Proapoptotic, proinflammatory, inhibits insulin } \\
\text { action }\end{array}$ \\
\hline IL-6 & $\begin{array}{l}\text { Hepatic glucose output, peripheral insulin } \\
\text { resistance }\end{array}$ \\
\hline Resistin & Hepatic glucose output, proinflammatory \\
\hline Adiponectin & Reduces steatosis, inhibits stellate cell activation \\
\hline Leptin & Stellate cell activation/fibrosis \\
\hline PAI-1 & Stellate cell activation/fibrosis \\
\hline Angiotensinogen & Stellate cell activation/fibrosis \\
\hline Fatty acid & Pro-inflammatory \\
\hline
\end{tabular}


Hepatic steatosis stimulates hepatocyte secretion of inflammatory cytokines e.g. IL6, that modulate the insulin sensitivity by themselves, as well as, peripheral insulin resistance which leads the liver for further injury. ${ }^{7}$ Hepatic stellate cell-derived resistin also contributes to insulin resistance by upregulating hepatocyte production of TNFá, IL-6, etc; that increase hepatic glucose output while inhibiting muscle insulin sensitivity. ${ }^{7}$ They also reported that both the TNF-á and resistin, the pro-inflammatory factors, promote dephosphorylation and inactivation of adenosyl monophosphate-kinase (AMPK) in turn activating the acetyl CoA carboxylase, which enhances hepatic lipid accumulation by increasing fatty acid biosynthesis and inhibiting mitochondrial b-oxidation. The TNF-á levels, which is also produced abundantly by immature adipocytes ${ }^{7}$ increased significantly from obese controls $(1.9 \pm 0.3 \mathrm{pg} / \mathrm{ml})$, simple steatosis $(2.9 \pm 0.9 \mathrm{pg} / \mathrm{ml})$ to NASH $(8.2 \pm 21.7 \mathrm{pg} / \mathrm{ml})$, with reciprocal relationship of adiponectin which decreased significantly $(\mathrm{p}<0.001)$ from obese controls $(10.2 \pm 7.4 \mathrm{ig} / \mathrm{ml})$, simple steatosis $(12.2 \pm$ $7.56 \mathrm{ig} / \mathrm{ml})$ to NASH $(6.7 \pm 6.5 \mathrm{ig} / \mathrm{ml})$ indicating potential role of TNF-á in NAFLD pathogenesis. ${ }^{27}$

Adiponectin, an adipokine, which antagonizes the effect of pro-inflammatory cytokines such as TNF-á and resistin ${ }^{7}$ was down regulated and the inflammatory markers including $\mathrm{C}$ reactive protein (CRP) and fibrinogen were up regulated in overweight people. ${ }^{14}$ So the increased adiponectin level inhibits the biological activity of TNF-á and resistin. ${ }^{7}$ High prevalence of NAFLD in overweight males were associated with lowest levels of adiponectin. ${ }^{14}$

\section{Immune Response}

The immune cells in the liver, hepatic macrophages (Kupffer cells) and natural killer T (NKT) cells play important roles in tissue inflammatory responses. ${ }^{7}$ The activated Kupffer cells result in the release of an array of inflammatory mediators, growth factors, and ROS. Kupffer cells are the sources of several cytokines like: IL-10, IL-12, transforming growth factor beta (TGF-b) 1, interferon (IFN)g, etc. that modulates the biological activity of TNF-á. ${ }^{28}$ The abnormalities of the hepatic immune system lead to the increased production of inflammatory cytokines which contribute to chronic inflammatory state of NAFLD. ${ }^{7}$ The activated hepatic stellate cells produce collagen matrix as a healing response which ultimately progress to fibrosis. ${ }^{7}$ Study in rats and mice showed that kupffer cells are responsible for phagocytosing gut-derived endotoxin to prevent its escape into the blood circulation becoming endotoxemia but when the kupffer cell specific gene is reduced the phagocytic activity of kupffer cell is also reduced making liver more vulnerable to endotoxin injury. ${ }^{28}$ In the same study LPS exposure in animals over expressed the IFNg mRNA, a hepatocyte sensitizer to TNF toxicity, and lowered the expression of IL10 and IL-12 mRNA, the TNF inhibitors, making the fatty rodents more vulnerable to TNF toxicity indicating that obesity is associated with abnormal kupffer cell function. ${ }^{28}$ Altered production of inflammatory mediators by kupffer cell ${ }^{7}$ and increased hepatocyte sensitivity to TNFá were the two underlying mechanisms for the increased hepatotoxicity. ${ }^{28}$

Kupffer cells are the predominant sources of ROS and their activation enhance the plasma concentrations of glutathione disulfide (GSSG; index of oxidant stress) or lower GSH contributing to ischemia-reperfusion injury and oxidative stress to the liver. ${ }^{29}$ Hepatic depletion of NKT cells, the lymphatic immune cells, is one of the immune system defects because it had been proved that replenished NKT cell population had reduced the inflammatory cytokine production and improved NAFLD. ${ }^{7}$

Generally, circulating IgG against lipid peroxidation products become significantly higher $(\mathrm{p}<0.001)$ in NAFLD patients than in controls. ${ }^{30}$ They also reported that the immune reactions triggered by oxidative stress can be an independent predictor of progression of NAFLD to advanced fibrosis. The anti-MDA antibody (lipid peroxidation related antibody) was significantly associated with progression of NAFLD. ${ }^{30}$

\section{Insulin resistance}

Insulin resistance is almost a universal finding in patients with NAFLD. ${ }^{8}$ It affects carbohydrate and fat metabolism increasing triglyceride accumulation (TG) accumulation in the liver. It is an important driving force of pathogenesis ${ }^{8}$ which promotes an accumulation of visceral adipose tissue and lipolysis of peripheral adipose tissue increasing FFA influx into the liver. ${ }^{1,2}$ Resistance to insulin's stimulation of glucose uptake via GLUT-4 by skeletal muscle and adipose tissue diverts the dietary glucose into the liver which is not insulin dependent. Insulin also stimulates de novo lipogenesis in the liver. All these consequences lead to increased TG accumulation in the liver which causes liver steatosis. $^{11}$

Different proinflammatory cytokines like; TNF-á; a cytokine that interferes with insulin-receptor mediated signal pathways in hepatocytes, IL-6, resistin, etc. inhibit the insulin action. ${ }^{31}$ They also reported that NAFLD was associated with insulin resistance and hyperinsulinemia even in lean subjects with normal glucose tolerance. Insulin mediated suppression of lipolysis was reported to be less effective in NAFLD patients (-69\%) compared to control subjects ($84 \%) .{ }^{32}$ They also reported that hepatic glucose production decreased by only $63 \%$ of basal in NAFLD compared to $84 \%$ in control subjects in response to insulin infusion. 


\section{Metabolic syndrome}

NAFLD may be considered an additional feature of the metabolic syndrome (Table 4), with specific hepatic insulin resistance.$^{32} \mathrm{It}$ is associated with many features of metabolic syndrome which includes central obesity, hypertriglyceridemia, low HDL-cholesterol, hypertension, hyperinsulinemia and hyperglycemia. ${ }^{8,33,34}$

Table 4: Clinical features of metabolic syndrome in 30 NAFLD patients. ${ }^{32}$

\begin{tabular}{|c|c|}
\hline Clinical features & $\begin{array}{l}\text { Prevalence in } \\
\text { percent } \\
(95 \% \text { CI })\end{array}$ \\
\hline \multicolumn{2}{|l|}{ Obesity } \\
\hline Overweight (BMI $25.0-29.9 \mathrm{~kg} / \mathrm{m}^{2}$ ) & $67(47-83)$ \\
\hline \multicolumn{2}{|l|}{ Central Fat Accumulation } \\
\hline \multicolumn{2}{|l|}{ Or $88 \mathrm{~cm}$ (women) } \\
\hline Hypertriglyceridemia (>2 mmol/L) & $47(28-66)$ \\
\hline Low HDL cholesterol (<1 ìmol/L) & $43(25-63)$ \\
\hline Hypertension (>160/95 mmHg) & $17(6-35)$ \\
\hline \multicolumn{2}{|l|}{ Impaired glucose tolerance } \\
\hline Increased fasting insulin $(>100 \mathrm{pmol} / \mathrm{L})$ & $57(38-75)$ \\
\hline Postload hyperinsulinemia (>1000 pmol/L & $27(12-46)$ \\
\hline
\end{tabular}

According to the ATP III criteria, the prevalence of metabolic syndrome in patients with NAFLD was $22.8 \%$ with increased liver enzymes (AST/ALT ratio >1) in $21.16 \%$ of the patients. ${ }^{35}$ They agreed on that NAFLD is associated with several metabolic disturbances with insulin resistance as a link between metabolic disturbances and NAFLD.

\section{Abnormal methionine metabolism}

Abnormal methionine metabolism (Fig. 2) causes deficiency of S-adenosyl methionine (SAMe), an intermediate and allosteric activator in the pathway of GSH synthesis, which ultimately leads to GSH deficiency. ${ }^{36}$

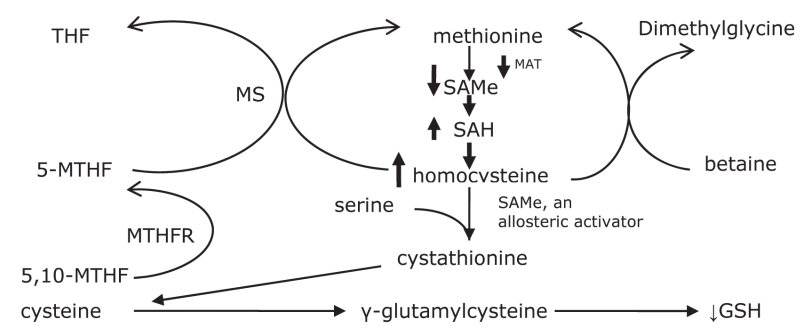

Fig. 2: Diagram of hepatic methionine metabolism. There is impaired conversion of methionine to s-adenosyl methionine (SAMe) because of decreased hepatic methionine adenosyltransferase activity which stimulates endotoxin-stimulated TNF production. However, there is an increased level of both s-adinosylhomocysteine (SAH) and homocysteine in many forms of fatty liver which sensitizes TNF hepatotoxicity. MS, methionine synthase; THF, tetrahydro folate

\section{Obesity related injury}

Obesity promotes lipopolysaccharide (LPS)-induced liver injury by sensitizing the hepatocyte to TNF toxicity. ${ }^{28}$ Based on animal model experiment they reported that fatty individuals are prone to endotoxin liver injury than lean ones. They found that $21 \pm 2 \%$ of the fatty rats were dead within 12 hours of LPS injection $\left(0.5 \mathrm{mg} \mathrm{kg}^{-1}\right)$ and rest of all surviving fatty rats had developed steatohepatitis with increased aminotransferase levels, but in contrast, LPS treated lean rats exhibited little toxicity and none of them neither died nor developed the evidence of steatohepatitis.

Several studies showed high prevalence of NAFLD in overweight and obese people than in lean individuals..$^{31,37,38}$ The significant of central obesity is more meaningful for NAFLD in the normal weight group than in the overweight group; and this suggests that visceral adiposity rather than the overall amount of body fat is important particularly in normal weight people. ${ }^{39}$

\section{Dietary factor}

Importantly, excessive carbohydrate intake and fat intake could play a role in increasing blood glucose, FFA, and insulin concentrations. ${ }^{11}$ They also reported that accumulation of saturated fatty acids (SFA) in the liver due to high SFA or high fructose diets lead to an increase in markers associated with endoplasmic reticulum stress and liver dysfunction. Hepatic steatosis is closely linked to consumption of high fructose corn syrup and certain types of fats. ${ }^{36}$ According to recent finding pathogenic mechanism underlying the development of NAFLD was somehow associated with excessive dietary fructose consumption when they found nearly 2 to 3 fold higher fructose level in the patients with biopsy proven NAFLD compared to their matched controls $\left(365 \mathrm{kcal} \mathrm{day}^{-1}\right.$ and $170 \mathrm{kcal} \mathrm{day}^{-1}$, respectively, $\mathrm{P}$ d" 0.05). ${ }^{33}$

Similarly, it is found lower intake of vitamin A and E in NAFLD patients compared to the persons without NAFLD and reduced intake of vitamin A was independently correlated with the severity of liver disease. ${ }^{25}$ Effect of diet was also seen in animal model of liver disease ${ }^{24}$ where methionine and choline deficient (MCD) diet fed mice developed steatosis, ballooning generation and lobular inflammation irrespective of dietary lipid composition. Serum ALT levels were increased by 10 fold ( $\mathrm{P}$ d" 0.001) in mice fed MCDdiets supplemented with $20 \%$ fat. ${ }^{24}$ But they also reported that mice fed olive oil MCD diet exhibited less elevation of serum ALT levels but ballooned hepatocytes was increased compared to lard MCD fed mice. 


\section{Nutrient gene interaction}

Genetic factors might be primarily responsible for increased serum triglyceride levels reducing the peripheral insulin sensitivity at a receptor level, however in NAFLD patients who were overweight, obese or who had type 2 diabetes, the increased serum triglyceride levels was due to enhanced peripheral lipolysis. ${ }^{31}$ It has been found that hepatic mRNA expression of fructokinase, an important and first enzyme for fructose metabolism, and fatty acid synthase, an important enzyme for lipogenesis, were both upregulated $(\mathrm{P}=0.04$ and $\mathrm{P}=0.02$, respectively) in NAFLD patients with high dietary fructose consumption compared to controls. ${ }^{33}$

Hepatic expression of lipogenic transcription factor, sterol regulatory element binding protein-1 (SREBP-1) was reduced while two enzymes involved in triglyceride synthesis, diacylglycerol acyltransferase -1 and -2 (DGAT-1 and -2) were increased in mice fed lard and olive oil diet compared to mice fed lipid-supplemented control diets, and were further increased 3 fold by MCD-feeding but neither of them prevented the accumulation of FFA in MCD-fed mice. ${ }^{24}$ So the nutritional model of steatohepatitis in mice showed that accumulation of FFA occurs despite substantial suppression of lipogenesis and induction of triglyceride synthesis genes. ${ }^{24}$

\section{Risk factors for NAFLD}

Major risk factors for NAFLD are diabetes, obesity, insulin resistance, hyperlipidemia, hyperglycemia, and older age. ${ }^{1,16}$ In the patients with NAFLD, 20\% had diabetes, 52\% had obesity and $38 \%$ had central obesity in a population based study in India. ${ }^{15}$ Cross-sectional studies done in China showed that gender (male), urban residency, low education, high blood pressure, diabetes, high BMI, high waist circumference, high waist to hip ratio, high serum triglyceride and glucose levels were the risk factors for FLD. ${ }^{13,40}$ It has been reported that the risk factors independently associated with NAFLD in Israeli subjects included male gender [odds ratio $(\mathrm{OR})=2.8,95 \%$ confidence interval $(\mathrm{CI})$ 1.5-5.3], abdominal obesity $(\mathrm{OR}=2.9,95 \%$ CI 1.3-6.4), hyperinsulinemia $(\mathrm{OR}=2.3,95 \% \mathrm{CI} 1.2-4.3, \mathrm{P}$ d" 0.01) and hypertriglyceridemia $(\mathrm{OR}=2.4,95 \% \mathrm{CI} 1.3-4.5) .{ }^{17}$

Another finding on population based study in the Philippines showed that the number of females was greater than males ( $71 \%$ and $29 \%$, respectively) in 134 NAFLD patients. ${ }^{37}$ Similarly, it has been reported that the prevalence rate of FLD was significantly higher in men than in women $42 \%$ in white men; $24 \%$ in white women ${ }^{18}$ under the age of 50 years $(22.4 \%$ and $7.1 \%$ respectively $\mathrm{P}$ d" 0.001$)$ but the opposite was found over the age of 50 years $(20.6 \%$ and $27.6 \%$ respectively, $\mathrm{P}$ d" $0.05 \%) .{ }^{13}$ Animal model of liver injury showed that females were more sensitive to TNF-á since they developed most endotoxin liver injury with lowest serum concentration of TNF-á. ${ }^{28}$

High prevalence of NAFLD with increased age, 65.4\% (4059 years) and $74.6 \%$ (e"60 years, P d" 0.001) among participants, was found in another study. ${ }^{12}$ But De Lusong et $\mathrm{al}^{37}$ found no significant relationship between presence of fatty liver and age in Philippine subjects.

\section{Treatment for NAFLD}

The improvement from an intervention is most readily achieved at the stage of simple steatosis and improvement in histology can be expected before the development of cirrhosis. $^{2}$ Once cirrhosis has developed, liver disease due to NAFLD can no longer be reversed. ${ }^{2}$ There are several pathophysiological effects of therapies that either reduce FFA production, or inhibit TNF-á production and its activity, or inhibit the suppression of adiponectin, or inhibit oxidative stress, triglyceride synthesis, hepatic stellate cell activation. ${ }^{47}$ Following approaches have been suggested for the management of patients with NAFLD: ${ }^{2}$

1) Correction of the underlying risk factors for NAFLD

2) Avoidance of alcohol consumption

3) Pharmacological treatment for NAFLD

\section{Correction of underlying risk factors}

NAFLD is often diagnosed in association with insulin resistance or metabolic syndrome, so treatment of risk factors is mandatory in patients with NAFLD. ${ }^{2,38}$ Optimal therapy of NAFLD appears to include lifestyle modification with exercise, diet and weight loss. ${ }^{36}$ Improvement in insulin resistance with lifestyle intervention (diet and increased physical activity) continues an essential step in both the treatment and prevention of NAFLD. ${ }^{8}$ Treatment of patients with NAFLD should focus on metabolic risk factors ${ }^{1}$ and management of associated conditions including obesity, blood glucose and lipid abnormalities (diabetes, dyslipidemia), hypertension, etc. ${ }^{2,8}$

\section{Nutritional therapy}

Dietary modification in an early stage may have a positive impact on the treatment of NAFLD. ${ }^{2}$ There are several diets to choose from those advocated by various medical associations (American Heart Association, American Diabetes Association, etc.) and commercial enterprises (Atkins, South Beach, etc.) to reduce the metabolic risk factors. ${ }^{2}$ US department of Agriculture (USDA) dietary guidelines recommend individuals to take an appropriate amount of calories for body size and activity level, variety of foods chosen from several food groups, whole grains and their products, more servings of fruits and vegetables, meats and dairy products. ${ }^{11}$ Vitamin $\mathrm{E}$ is a potent chain 
breaking antioxidant that is widely used as a nutritional supplement, since patients with various types of liver diseases frequently have low serum vitamin E concentrations. ${ }^{36}$ They also reported that vitamin $\mathrm{E}$ has been shown to attenuate a variety of experimental liver injury, to reduce endotoxin or LPS-stimulated TNF production and to block stellate cell activation. SAMe, a precursor of GSH, is also available as a nutritional supplement in some countries. ${ }^{36}$ They also reported that betaine can be given to reduce the homocysteine and $\mathrm{SAH}$ levels generating methionine. Supplementation with ù-3 and ù-6 long chain PUFAs along with an appropriate antioxidant may be of benefit for NAFLD. ${ }^{26,41}$ PUFAs ameliorate hepatic steatosis by down regulation of lipogenic transcription factor; SREBP1 and activation of perioxisome proliferator activated receptor alpha (PPARá); a mediator of peroxisomal and microsomal fatty acid oxidation. ${ }^{41}$

\section{Weight Reduction and Physical Exercise}

Since obesity is the common risk factor for NAFLD, weight reduction plays an important role in treatment of NAFLD. ${ }^{2}$ Basically, gradual weight loss and physical exercise can be the gold standard for all forms of NAFLD. ${ }^{11}$ Weight reduction by at least $5 \%$ with subsequent weight control and regular exercise may be beneficial in treating NAFLD. ${ }^{42}$ But weight reduction is not always an effective treatment because it sometimes, may exacerbate the disease. ${ }^{43}$

Pharmacologic treatment of obesity may be considered in individuals with a BMI $>30 \mathrm{~kg} \mathrm{~m}^{-2}$ or a BMI $>27 \mathrm{~kg} \mathrm{~m}^{-2}$ plus associated obesity-related comorbidities and bariatric surgery may be considered for those with morbid obesity i.e; $\mathrm{BMI}>40 \mathrm{~kg} \mathrm{~m}^{-2} .2,8$ The proof of steatohepatitis or fibrosis on liver biopsy can affect the decision whether to proceed for a more aggressive therapeutic option, i.e; bariatric surgery or an experimental pharmacologic treatment for NAFLD. ${ }^{2}$

\section{Pharmacological therapy for NAFLD}

There is no effective medical therapy currently available for all patients with NAFLD. ${ }^{1,19}$ Pharmacological treatment of NAFLD (Table 5) includes weight loss, modulation of insulin resistance and reduction of inflammation. ${ }^{2}$ Rapid weight loss may worsen NASH, so use of other medications that can directly reduce or reverse liver damage independent of weight loss, is a reasonable alternative. ${ }^{19}$ They also pointed out that pharmacological therapy may be of particular benefit for patients who do not lose weight or cannot maintain long term weight reduction. Medications that increase the insulin sensitivity and antioxidant defenses help the treatment of NAFLD patients, who fail to or do not need to lose weight. ${ }^{8,25}$ Hepatoprotective and insulin sensitizing drugs are being used in trials but no proven effect has been demonstrated to prevent the disease progression. ${ }^{1}$

In vitro experiments and animal studies showed that PPARá discloses anti-inflammatory property and PPAR ã discloses anti-inflammatory, anti-fibrogenic and antiproliferative properties in the liver. ${ }^{44}$ Thiazolidinediones (TZDs) are high-affinity ligands of PPAR-ã, which improves the insulin sensitivity by stimulating the storage of FFA in the subcutaneous adipocytes and the liver through their action as PPAR- ã agonists that increases plasma adiponectin levels and fatty acid oxidation and decreases fatty acid synthesis. ${ }^{45,46}$ Metformin improves insulin resistance by decreasing hepatic glucose production and increasing skeletal muscle glucose uptake. It also reduces hepatic expression of TNF-á, suppress lipogenesis and increase fatty acid oxidation. ${ }^{47,48}$ Similarly, statins (hydroxymethylglutoryl-CoA reductase inhibitors) are a class of highly prescribed medications used to treat hyperlipidemia, and there has been concern regarding their use in patients with fatty liver disease. ${ }^{49}$

SREBPs are major transcriptional regulators of cholesterol, fatty acid and glucose metabolism. Genetic disruption of SREBP activity reduces plasma and liver levels of cholesterol and triglycerides as well as insulin stimulated lipogenesis, suggesting that SREBP is an important target for pharmacological intervention. ${ }^{50}$ Liver steatosis and fibrosis were decreased ( $\mathrm{P} \mathrm{d} \leq 0.05)$ withoutthe improvement in lobular inflammation or hepatocellular ballooning in patients with biopsy-proven NASH enrolled in a medical therapy orally fed with $\mathrm{N}$-acetylcysteine and metformin. ${ }^{51}$

Table 5: Pharmacological therapies in the treatment of NAFLD. ${ }^{47}$

\begin{tabular}{|c|c|c|c|}
\hline Treatment & Mechanism & Biochemical effect & Histological effect \\
\hline Orlistat & Weight loss & $\begin{array}{l}\downarrow \text { LFTs and insulin } \\
\text { resistance }\end{array}$ & $\begin{array}{l}\downarrow \text { steatosis, } \\
\text { inflammation }\end{array}$ \\
\hline Rimonabant & $\begin{array}{l}\text { Weight loss, } \\
\text { peripheral effects }\end{array}$ & $\begin{array}{l}\downarrow \text { insulin resistance, TG } \\
\text { levels and LFTs }\end{array}$ & $\downarrow$ steatosis \\
\hline TZDs & PPAR- $\gamma$ agonists & $\begin{array}{l}\downarrow \text { LFTs, insulin resistance } \\
\text { and TNF- } \alpha\end{array}$ & $\begin{array}{l}\downarrow \text { steatosis, inflammation } \\
\text { and fibrosis }\end{array}$ \\
\hline Metformin & $\uparrow$ AMP kinase & $\begin{array}{l}\downarrow \text { LFTs, } \quad \text { and } \quad \text { insulin } \\
\text { resistance }\end{array}$ & $\begin{array}{l}+/ \text {-improvement in } \\
\text { steatosis, inflammation and } \\
\text { fibrosis }\end{array}$ \\
\hline Vitamin $E$ & $\downarrow$ oxidative stress & $\downarrow$ LFTs & Uncertain \\
\hline Betaine & \oxidative stress & $\downarrow$ LFTs & $\begin{array}{l}\downarrow \text { steatosis, inflammation } \\
\text { and fibrosis }\end{array}$ \\
\hline Pentoxifylline & Hepatoprotective & $\downarrow$ LFTs and TNF $\alpha$ & $\downarrow$ steatosis and inflammation \\
\hline
\end{tabular}

Notes: “!, decreases; '!, increases; AMP, adenosine 5'monophosphate; LFT, liver function test; PPAR, perioxisome proliferator-activated receptor; TNF, tumor necrosis factor; TZD, thiazolidinediones. 


\section{Avoidance of alcohol consumption}

It is considered that up to two alcoholic drinks ( $40 \mathrm{gm} /$ day) for male and one drink (20 gm/day) for female is not associated with clinically significant liver damage. ${ }^{2,3}$ It is better for the patients with NASH not to drink alcohol to minimize the disease progression. ${ }^{2,8}$

\section{Liver transplantation}

A liver transplant is surgery to replace a diseased liver with a portion of or a complete healthy liver. ${ }^{3}$ Liver transplantation is a life-extending therapeutic alternative for patients with end-stage liver disease but NAFLD may recur even after liver transplantation. ${ }^{19}$

\section{Discussion}

This study provides an in-depth analysis of mechanisms of NAFLD development, progression and therapies. Although the exact prevalence of NAFLD in general population has not yet been determined, it has been quoted around $20 \%$ in different countries ${ }^{10}$ and almost $30 \%$ in the United States..$^{9,11}$ There is a modern epidemic of obesity, metabolic syndrome, and diabetes; risk factors for NAFLD, in developed countries and people with western lifestyle in the developing countries. NAFLD can progress to cirrhosis overtime in some individuals but not in all. NAFLD is associated with an increase in lipogenesis, a defective mitochondrial and peroxisomal b-oxidation and/or diminished ability of the liver to export lipids. ${ }^{26}$

The first 'hit' according to the prevailing 'two-hit' hypothesis ${ }^{23}$ to the pathogenesis of NAFLD, is peripheral and hepatic insulin resistance leading to increased lipolysis in adipose tissue, delivery of FFA, de novo synthesis of fatty acids in liver and development of steatosis. The second 'hit' is caused by oxidative stress and abnormal cytokine production leading to lipid peroxidation, inflammation and fibrogenesis. Majority of the cases of raised liver enzymes in T2DM patients is due to NAFLD. ${ }^{20}$

Altered adipokine signaling and oxidative stress balance have been implicated in the pathogenesis of both NAFLD and insulin resistance. ${ }^{25}$ Insulin resistance and the metabolic syndrome have been associated with NAFLD, and the number and severity of features of the syndrome predict the severity of liver disease. ${ }^{25}$ NAFLD is characterized by a remarkable reduction of insulin sensitivity, with decreased effects of insulin on both glucose and lipid metabolism. ${ }^{32}$ Such a defect is not only associated with the abnormal glucose regulation and/or overweightness, as observed frequently in NAFLD, but also with normal glucose tolerance and lean subjects. Both during fasting and in response to glucose ingestion, the liver itself might be responsible for insulin resistance since it does not switch off glucose production. ${ }^{32}$ NAFLD patients, even in the presence of normal body weight, have a relatively increased visceral adiposity, which might be relevant in the pathogenesis of liver injury. ${ }^{32}$

The TNF-á has potential role in pathogenesis of NAFLD as its level increases significantly from obese controls, simple steatosis to $\mathrm{NASH} .{ }^{27}$ Accumulation of FFA supports a lipotoxicity mechanism for liver injury. ${ }^{24}$ Several studies suggest that excessive fructose intake is a risk factor for developing NAFLD. ${ }^{36}$ Poor dietary habits also may have contributed to oxidative stress in the patients because they consumed lower amounts of antioxidant vitamins compared to control subjects. ${ }^{25}$

Kupffer cells are resident macrophages of the liver and play an important role in its normal physiology and homeostasis as well as participating in the acute and chronic responses of the liver to toxic compounds. ${ }^{29}$ Altered production of inflammatory mediators by kupffer cell ${ }^{7}$ and increased hepatocyte sensitivity to TNFá were the two underlying mechanisms for the increased hepatotoxicity. ${ }^{28}$ The abnormalities of the hepatic innate immune system (macrophages, neutrophils, natural killer T cells) lead to the increased production of inflammatory cytokines which contribute chronic inflammatory state of liver injury. ${ }^{7}$

Treatment of risk factors is mandatory in NAFLD patients ${ }^{2,38}$ including lifestyle modification with diet, exercise, and weight loss. ${ }^{36}$ PPAR agonists and disruption of SREBP activity are important targets for pharmacological intervention of fatty liver disease. ${ }^{45,46,50}$ Pentoxifylline, a TNF-á inhibitor, treatment resulted in a significant reduction of serum TNF-á level accompanied by normalization of both ALT and AST, and improvements in the insulin resistant index indicating the potential role of anti-TNFá in the treatment of liver injury. ${ }^{27}$

\section{Conclusions}

NAFLD has become a significant public health problem which is associated with insulin resistance and metabolic syndrome. It is prevalent in patients with T2DM and raised liver enzymes. Clinical studies have established a spectrum of NAFLD from simple steatosis to steatohepatitis, fibrosis, cirrhosis, hepatocellular carcinoma and liver failure. But bland steatosis, sometimes, remains stable for a number of years and will probably never progress in many cases. With the increasing prevalence of obesity, T2DM, and the metabolic syndrome in the general population NAFLD has become a common diagnosis in clinical practice. Excess 
production of cytokines and ROS; and shortage of GSH, and adiponectin production play key roles in the pathogenesis of liver injury. Immune system becomes defective and anti-inflammatory cytokines and antioxidants are unable to overcome inflammation and fibrosis in progressive liver injury.

There is no consensus as to what diet and lifestyle approach is the right one for NAFLD patients because of the lack of scientific evidence. Standard guidelines for the therapy of NAFLD continue to be work in progress with weight loss and lifestyle modification being the mainstays of therapy. More studies are needed to clarify the specific effects of different diets and dietary components on the health of NAFLD patients. Additional research is required to determine optimal therapies to prevent and recover from NAFLD.

\section{References}

1. Adams LA, Lindor KD. Nonalcoholic fatty liver disease. Ann Epidemiol 2007 Nov;17(11):863-869.

2. Yan E, Durazo F, Tong M, Hong K. Nonalcoholic fatty liver disease: pathogenesis, identification, progression, and management. Nutr Rev 2007 Aug;65(8):376-384.

3. American Liver Foundation. WWW document. URL: http://www.liverfoundation.org./education/info/ accessed on June 1, 2008.

4. Ludwing J, Viggiano TR, McGill DB, Oh BJ. Nonalcoholic steatohepatitis: Mayo Clinic experiences with a hitherto unnamed disease. Mayo Clin Proc 1980 Jul;55(7):434-438.

5. Denk H, Stumptner C, Fuchsbichler A, Zatloukal K. Alcoholic and non-alcholic steatohepatitis. Verh Dtsch Gcs Pathol 2005;89:137-143.

6. Kazemi-Shirazi L, Veloso MP, Frommlet F. Differentiation of nonalcoholic from alcoholic steatohepatitis: are routine laboratory markers useful? Wien Klin Wochenschr 2008;120(1-2):25-30.

7. Yang L, Diehl AM. Role of immune response in nonalcoholic fatty liver disease: evidence in human and animal studies. In: Gershwin ME, Vierling JM, Manns MP, ed. Liver immunology: principles and practice. Totowa: Humana Press, 2007;337-345.

8. Angulo P. Obesity and nonalcoholic fatty liver disease. Nutr Rev 2007 Jun;65 (6):57-63.

9. Rector RS, Thyfault JP, Wei YZ, Ibdah JA. Nonalcoholic fatty liver disease and the metabolic syndrome: an update. World J Gastroenterol 2008 Jan;14(2):185-192.

10. Joy D, Thava VR, Scott B. Diagnosis of fatty liver disease: is biopsy necessary? Eur J Gastroenterol Hepatol 2003 Mar;15(5):539-543.

11. Zivkovic AM, German JB, Sanyal AJ. Comparative review of diets for the metabolic syndrome: implications for nonalcoholic fatty liver disease. Am J Clin Nutr 2007 Aug;86(2):285-300.

12. Targher G, Bertolini L, Padovani R, Rodella S, Tessari $\mathrm{R}$, Zenari L, et al. Prevalence of nonalcoholic fatty liver disease and its association with cardiovascular disease among type 2 diabetic patients. Diabetes Care 2007 May;30(5):1212-1218.

13. Zhou YJ, Li YY, Nie YQ, Ma JX, Lu LG, Shi SL, et al. Prevalence of fatty liver disease and its risk factors in the population of South China. World J Gastroenterol 2007;13:6419-6424.

14. Imhof A, Kratzer W, Boehm B, Meitinger K, Trischler G, Steinbach G, et al. Prevalence of non-alcoholic fatty liver and characteristics in overweight adolescents in the general population. Eur J Epidemiol 2007 Sep;22:889-897.

15. Amarapurkar D, Kamani P, Patel N, Gupte P, Kumar P, Agal S, et al. Prevalence of nonalcoholic fatty liver disease: population based study. Ann Hepatol 2007 Jul-Sep;6(3):161-163.

16. Park SH, Jeon WK, Kim SH, Kim HJ, Park DI, Cho YK, et al. Prevalence and risk factors of nonalcoholic fatty liver disease among Korean adults. J Gastroenterol Hepatol 2006;21(1):138-143.

17. Zelber-Sagi S, Nitzan-Kaluski D, Halpern Z, Oren R. Prevalence of primary non-alcoholic fatty liver disease in a population based study and its association with biochemical and anthropometric measures. Liver Int 2006;26 (7):856-863.

18. Browning JD, Szczepaniak LS, Dobbins R, Nuremberg P, Horton J, Cohen J, et al. Prevalence of hepatic steatosis in an urban population in the United States: impact of ethnicity. Hepatology 2004 Dec;40(6):1387395.

19. Angulo $P$, Lindor KD. Non-alcoholic fatty liver disease. J Gastroenterol Hepatol 2002 Feb;17:186-190.

20. Hickman IJ, Russell AJ, Prins JB, Macdonald GA. Should patient with type 2 diabetes and raised liver enzymes be referred for further evaluation of liver 
disease? Diabetes Res Clin Prac 2008 Apr;80(1):e10e12.

21. Jimba S, Nakagamit T, Takahashi M, Wakamatsu T, Hirota Y, Iwamotot Y. Prevalence of nonalcoholic fatty liver disease and its association with impaired glucose metabolism in Japanese adults. Diabet Med 2005 Sep;22(9):1141-1145.

22. Hasse JM, Matarese LE. Medical nutrition therapy for liver, biliary system, and exocrine pancreas disorders, $11^{\text {th}}$. ed. In: Mahan LK, Escott-Stump S, ed. Krause's food, nutrition and diet therapy. Philadelphia, Elsevier, 2004; 738-767.

23. Day CP, James OF. Steatohepatitis: a tale of two "hits"? Gastroenterology 1998 Apr;114(4):842-845.

24. Larter CZ, Yeh MM, Haigh WG, Williams J, Brown S, Bell-Anderson KS, et al. Hepatic free fatty acids accumulate in experimental steatohepatitis: role of adaptive pathways. J Hepatol 2008 Apr;48(4):638-647.

25. Musso G, Gambino R, De Michieli F, Biroli G, Premoli A, Pagano G, et al. Nirosative stress predicts the presence and severity of nonalcoholic fatty liver at different stages of the development of insulin resistance and metabolic syndrome: possible role of vitamin A intake. Am J Clin Nutr 2007 Sep;86(3):661-671.

26. Allard JP, Aghdassi E, Mohammed S, Raman M, Avand G, Arendt BM, et al. Nutritional assessment and hepatic fatty acid composition in non-alcoholic fatty liver disease (NAFLD): a cross-sectional study. $J$ Hepatol 2008 Feb;48(2):300-307.

27. Jarrar MH, Baranova A, Collantes R, Ranard B, Stepanova M, Bennett C, et al. Adipokines and cytokines in non-alcoholic fatty liver disease. Aliment Pharmacol Ther 2008 Mar;27(5):412-421.

28. Yang SQ, Lin HZ, Lane MD, Clemens M, Diehl AM. Obesity increases sensitivity to endotoxin liver injury: Implications for the pathogenesis of steatohepatitis. Proc Natl Acad Sci 1997 Mar;94(6):2557-2562.

29. Jaeschke H, Farhood A. Neutrophil and kupffer cellinduced oxidant stress and ischemia-reperfusion injury in rat liver. Am J Physiol Gastrointest Liver Physiol 1991;260 (3):355-362.

30. Albano E, Mottaran E, Vidali M, Reale E, Saksena S, Occhino G, et al. Immune response towards lipid peroxidation products as a predictor of progression of non-alcoholic fatty liver disease to advanced fibrosis. Gut 2005 Jul;54(7):987-993.
31. Marchesini G, Brizi M, Morselli-Labate AM, Bianchi G, Bugianesi E, McCullough AJ, et al. Association of nonalcoholic fatty liver disease with insulin resistance. Am J Med 1999 Nov;107(5):450-455.

32. Marchesini G, Brizi M, Bianchi G Tomassetti S, Bugianesi E, Lenzi M, et al. Nonalcoholic fatty liver disease: a feature of the metabolic syndrome. Diabetes 2001 Aug;50(8):1844-1850.

33. Ouyang X, Cirillo P, Sautin Y, McCall S, Bruchette JL, Diehl AM, et al. Fructose consumption as a risk factor for non-alcoholic fatty liver disease. J Hepatol 2008 Mar;48(6):993-999.

34. Bedogni G, Miglioli L, Masutti F, Tiribelli C, Marchesini G, Bellentani S. Prevalence of and risk factors of nonalcoholic fatty liver disease: the dionysos nutrition and liver study. Hepatology 2005 Jul;42(1):44-52.

35. Lizardi-Cervera J, Lapara DI, Chavez-Tapia NC, Ostos ME, Esquivel MU. Prevalence of NAFLD and metabolic syndrome in asymptomatics subjects. Rev Gastroenterol Mex 2006;71(4):453-459.

36. Cave M, Deaciuc I, Mendez C, Song Z, Joshi-Barve S, Barve S, et al. Nonalcoholic fatty liver disease: predisposing factors and the role of nutrition. $J$ Nutr Biochem 2007;18(3):184-195.

37. De Lusong MAA, Labio E, Daez L, Gloria V. Nonalcoholic fatty liver disease in Philippines: Comparable with other nations? World J Gastroenterol. 2008 Feb;14(6):913-917.

38. Goenka N, Subramanian S, Weston P, Vora J. Nonalcoholic fatty liver disease: more than "just a bit of fatty liver". Practical Diabetes Int 2007 JulAug;24(6):305-308.

39. Kim HJ, Kim HJ, Lee KE, Kim DJ, Kim SK, Ahn CW, et al. Metabolic significance of nonalcoholic fatty liver disease in non obese, non diabetic adults. Arch Intern Med 2004 Oct;164(19):2169-2175.

40. Fan JG, Zhu J, Li XJ, Chen L, Li L, Dai F, et al. Prevalence of and risk factors for fatty liver in a general population of Shanghai, China. J Hepatol 2005 Sep;43(3):508-514.

41. Sekiya M, Yahagi N, Matsuzaka T, Najima Y, Nakakuki M, Nagai R, et al. Polyunsaturated fatty acids ameliorate hepatic steatosis in obese mice by SREBP-1 suppression. Hepatology 2003 Dec;38:1529-1539.

42. Suzuki A, Lindor K, St Saver J, Lymp J, Mendes F, Muto A, et al. Effect of changes on body weight and lifestyle in nonalcoholic fatty liver disease. $J$ Hepatol 


\section{Liver injury}

2005 Dec;43(6):1060-1066.

43. Tilg H, Diehl AM. Cytokines in alcoholic and non alcoholic steatohepatitis. New Eng J Med 2000;343:1467-1476.

44. Dharancy S, Louvet A, Hollebecque A, Desreumaux P, Mathurin P, Dubuquoy L. Nuclear receptor PPAR and hepatology: pathophysiological and therapeutical aspects. Gastroenterol Clin Biol 2008 Mar;32(3):339350.

45. Shulman GI. Cellular mechanisms of insulin resistance. J Clin Invest $2000 \mathrm{Jul} ; 106(2): 171-176$.

46. Lutchman G, Promrat K, Kleiner DE, Heller T, Ghany MG, Yanovski JA, et al. Changes in serum adipokine levels during pioglitazone treatment for nonalcoholic steatohepatitis, relationship to histologic improvement. Clin Gastroenterol Hepatol 2006 Aug;4(8):1048-1052.

47. Torres DM, Harrison SA. Diagnosis and therapy of nonalcoholic steatohepatitis. Gastroenterology 2008 May;134(6):1682-1698.

48. Zhou G, Myers R, Li Y, Chen Y, Shen X, Fenyk-Melody $\mathrm{J}$, et al. Role of AMP-activated protein kinase in mechanism of metformin action. J Clin Invest 2001 Oct;108(8):1167-1174.

49. Oh MK, Winn J, Poordad F. Review article: Diagnosis and treatment of nonalcoholic fatty liver disease. Aliment Pharmacol Ther 2008, in press, Epub (accessed on 15 June 2008).

50. Hawkins JL, Robbins MD, Warren LC, Xia D, Petras SF, Valentine JJ, et al. Pharmacological inhibition of site-1 protease activity inhibits SREBP processing and reducing lipogenic enzyme gene expression and lipid synthesis in cultured cells and experimental animals. $J$ Pharmacol Exp Ther 2008 Sep;326(3):801-808.

51. De Oliveira CP, Stefano JT, de Siqueira ER, Silva LS, de Campos Mazo DF, Lima VM, et al. Combination of Nacetylcysteine and metformin improves histological steatosis and fibrosis in patients with non-alcoholic steatohepatitis. Hepatol Res 2008;38(2):159-165. 